\section{Clinical characterization of brain tissue for neuroscience research: a comparison of antemortem and postmortem diagnoses}

\author{
N Sundquist', D Sheedy ${ }^{2}$, T Garrick ${ }^{2,3}$ \\ 'Neuroscience Institute of Schizophrenia and Allied Disorders (NISAD); \\ 2Discipline of Pathology, The University of Sydney, Australia; and \\ ${ }^{3}$ New South Wales Tissue Resource Centre, Sydney, Australia
}

Background: The validity of postmortem human brain research relies upon accurate clinical and psychopathological diagnosis. Current literature shows few instances where standardized diagnostic assessment tools such as the Diagnostic Instrument for Brain Studies (DIBS) have been used. The present study investigates the degree of concordance between predominant antemortem psychiatric diagnoses indicated in medical records and postmortem diagnoses derived through structured diagnostic instruments such as the DIBS and the Item Group Checklist (IGCL) of the Schedules for Clinical Assessment in Neuropsychiatry (SCAN).

Methods: Fifty-eight subjects from the New South Wales Tissue Resource Centre with a recorded diagnosis of mental illness during their lifetime were included in the study. The predominant antemortem psychiatric diagnosis of each case was compared with its corresponding postmortem diagnosis obtained through structured case reviews to which either the DIBS or the IGCL of the SCAN were applied. Demographic variables such as age of illness onset, and alcohol or other drug use were also examined.

Results: Comparison of diagnoses obtained from these two approaches produced an overall kappa coefficient of 0.66. Kappa coefficients for the schizophrenia cohort were $0.61,0.35$ for the schizoaffective cohort, 0.95 for the major depressive disorder cohort and 0.70 for the bipolar disorder cohort.

Conclusions: These results indicate moderate to excellent interrater reliability for most cohorts in this sample. There is sufficient disagreement, however, particularly in the schizoaffective cohort, to suggest the value of applying standardized and structured assessment to enhance both the accuracy of diagnosis and the prospective validity of tissue-based research.

\section{Delirium outcomes: is this a time-limited disorder?}

\section{J Symon}

University of Adelaide, Adelaide, Australia
Background: Delirium has classically been defined in relation to dementia, at least in its temporal dimension, delirium being a short-term condition, dementia long term. From the earliest accounts in the ancient medical literature to the contemporary DSM-IV-TR definition, it has been conceptualized as a time-limited process. It is also firmly established in the literature that delirium is underrecognized.

Methods: A literature search was undertaken using PubMed database covering the years 1966-2006 and using search terms that included delirium, organic brain syndrome, outcome, subsyndromal and identification.

Results: When compared with patients who did not suffer from delirium, delirium is associated with significantly greater mortality, higher rates of long-term cognitive impairment, greater length of stay and increased rates of institutional placement. These poor outcomes appear to hold for subsyndromal delirium as well as frank delirium. It is plausible to suggest that unrecognized delirium is associated a fortiori with poor outcomes, but as yet there is no firm evidence that early detection results in better outcomes.

Conclusions: The definition of delirium as a shortterm condition needs to be reevaluated. It could be speculated that its conceptualization as time limited may contribute to it's underrecognition because there may a little risk associated with missing a condition that is expected to resolve. In this case, it is expected that improved education for health professionals about the serious consequences and long-term course of delirium may result in improved detection and management of this disorder.

\section{Mismatch negativity in schizophrenia: effect of probability, deviant type and duration of illness}

\author{
J Todd ${ }^{1,2}$, PT Michie ${ }^{1,2}$, U Schall2,3, F Karayanidis ${ }^{1,2}$ \\ 'School of Behavioural Sciences, University of Newcastle; ${ }^{2}$ Neuroscience Institute \\ of Schizophrenia and Allied Disorders (NISAD); and ${ }^{3}$ Centre for Mental Health \\ Studies, University of Newcastle, Australia
}

Background: A reduced amplitude mismatch negativity (MMN) component of the auditory event-related potential (ERP) is repeatedly observed in individuals with schizophrenia. MMN amplitude also declines significantly with age in healthy adults. Our group is endeavoring to understand whether the factors underlying MMN reduction are common or unique. In this study, we explored the effect of deviant probability on group differences.

Methods: ERPs were recorded from 43 individuals diagnosed with schizophrenia and 42 age-matched 